\title{
L'ÉCRITURE SECRÈTE DE GERBERT.
}

